\title{
NEURAL NETWORK-BASED ADAPTIVE TRACKING CONTROL FOR A NONHOLONOMIC WHEELED MOBILE ROBOT SUBJECT TO UNKNOWN WHEEL SLIPS
}

\author{
TINH NGUYEN ${ }^{1}$, HUNG LINH LE ${ }^{2}$ \\ ${ }^{1}$ Institute of Information Technology, Viet Nam Academy of Science and Technology \\ ${ }^{2}$ University of Information and Communication Technology, Thai Nguyen University \\ ${ }^{1}$ nvtinh@ioit.ac.vn; ${ }^{2}$ lhlinh@ictu.edu.vn
}

\begin{abstract}
In this paper, Lagrange formula is employed with the purpose of modelling both the kinematics and dynamics of a nonholonomic wheeled mobile robot (WMR) subject to unknown wheel slips, model uncertainties such as unstructured unmodelled dynamic components, and unknown external disturbances such as unknown external forces. Afterwards, an adaptive tracking controller based on a radial basis function neural network (RBFNN) with an online weight tuning algorithm is proposed for tracking a predefined trajectory. Prior neural network offline training is not needed for the weights since they are easily initialized. Thanks to this proposed control approach, a desired tracking performance is obtained in which position tracking errors uniformly ultimately converge to an arbitrarily small neighborhood of the origin. In the sense of Lyapunov and LaSalle extension, the stability of the whole closed-loop system is guaranteed to achieve this desired tracking performance. The comparative results of computer simulation have validated the rightness and efficiency of the proposed controller.
\end{abstract}

Keywords. Online weight tuning algorithm, wheeled mobile robot, uniformly ultimately bounded, unknown wheel slip.

\section{INTRODUCTION}

It is well known that wheeled mobile robots (WMR) have ability to work in a wide area, and furthermore they are capable of performing tasks intelligently without any human action. Besides, they can replace people on dangerous tasks such as looking for explosive materials, transporting of goods in poisonous environments, rescue, etc. Therefore, they have been applied widely and increasingly popular in various areas such as industry, entertainment, health care, automation in logistics, transport, etc.

In recent years, many researchers in the world have paid their attention to research motion control problems for WMRs. In $[3,4,5,7,8]$, the controllers were designed to take account of kinematic and dynamic model of WMR without slipping motions.

Nonetheless, unfortunately, the condition "pure rolling without slip" has been not always satisfied in practice. To put it simply, there has been the existence of wheel slip. The wheel slip depends on a number of various factors namely an unknown centrifugal force acting on a WMR when it moves in a circular path, an external force acting on a WMR when it collides with another unknown object, a weak frictional force between the slippery floor and the wheels, etc. Consequently, if one wants 
a motion control problem to be effectively addressed in such context, then during designing motion controllers for WMR, the wheel slip must be considered. With the aim of compensating for the harmful effect of the wheel slip, an adaptive tracking controller has been developed via slip-ratios [9]. The approaches based on gyros and accelerometers to cope with the wheel slip have been also illustrated in $[2,13]$. The authors in [15] have illustrated the models of WMRs taking account of both wheel longitudinal and lateral slippage and then analyzed their controllability according to their maneuverability. Approaches for designing controllers have also been investigated in $[10,11]$ for the path following and tracking of WMRs in the presence of longitudinal and lateral slippage. In [17], S. J. Yoo has designed a neural-network-based adaptive control method for tracking path and avoiding obstacle for a class of WMRs in the presence of unknown skidding, slipping, and torque saturation. The work in [12] has addressed the slippage phenomenon for exactly kinematic modeling and then controlling for a WMR. In [6], the slip ratios of all wheels could be estimated via an experimental study. The authors in [14] have proposed a feedback linearization controller for a WMR tracking a desired trajectory with longitudinal and lateral slip under an ideal condition where there did not exist both model uncertainties and unknown external disturbances, and further the accelerations and velocities of the wheel slips were measured exactly. However, it is impossible to achieve a desired tracking performance in real applications because the ideal condition is not realistic.

To sum up, most of these aforementioned works except for $[1,16]$ have been based on an assumption that the measurements of the wheel slips were ready so as to analyze and design slip-compensation controllers. The drawback of this assumption is the extra demand of expensive and complex sensors to measure the wheel slip namely global position system (GPS), gyroscope, accelerometer, etc.

These results have motivated us to propose a novel neural network-based adaptive controller for a WMR with the both longitudinal and lateral wheel slip in such a way that the WMR tracks a desired trajectory with a desired tracking performance. Moreover, those measurements of wheel slip are no longer necessary.

The main purpose of this paper is that a neural network (NN) adaptive tracking controller is proposed for a WMR in the presence of unknown wheel slip, model uncertainties, and unknown external disturbances to track a predefined trajectory. Firstly, to do this, the Lagrange dynamic approach has been used to derive both the kinematics and the dynamics of the WMR in this situation. Secondly, with purpose of overcoming the harmful effect of unknown wheel slip, model uncertainties, and unknown external disturbances, a RBFNN adaptive tracking controller has been proposed. In this controller, the RBFNN with online weight tuning algorithm is employed to approximate unknown nonlinear smooth functions due to no prior knowledge of the dynamic model of this WMR. Finally, a Matlab/Simulink simulation was implemented to certify the effectiveness and the performance of the proposed controller.

The remainder of this article is structured as follows. Section 2 represents the progress by which both the kinematics and the dynamics of a WMR are modeled in the presence of both lateral and longitudinal slip between the driving wheels and the floor. In section 3, a RBFNN adaptive tracking controller with an online weight tuning algorithm is proposed, and the uniformly ultimately bounded stability of the closed-loop system to an adjustable neighborhood of the origin is proven in Lyapunov theory and LaSalle extension. Next, a computer simulation is shown in section 4 to certify the effectiveness and the performance of the proposed controller. Finally, section 5 illustrates our research conclusions. 


\section{THE KINEMATICS AND DYNAMICS OF A NONHOLONOMIC WMR WITH UNKNOWN WHEEL SLIP}

\subsection{The kinematic model}

Let us consider a nonholonomic WMR which comprises two driving wheels and a caster wheel as Figure 1. To be specific, $\mathrm{G}$ is the center of mass of the platform of the WMR. $M$ is the midpoint of the wheel shaft. $F_{1}$ and $F_{2}$ are the total longitudinal friction forces at the right and left wheel, respectively. $F_{3}$ is the total lateral friction force along the wheel shaft. $F_{4}$ and $\varpi$ are external force and moment acting on G, respectively. $r$ is the radius of each wheel. $b$ is the haft of the wheel shaft. $a$ is the distance between $M$ and $G$.

Let $x_{M}, y_{M}$ denote the coordinates of $M$. Likewise, let $x_{G}, y_{G}$ denote those of $G$. $\theta$ is the orientation of the WMR. When there does not exist wheel slip between the wheels and the floor, the linear and angular velocities of the WMR, computed at $\mathrm{M}$, are represented respectively as follows [8]

$$
\Theta=\frac{r\left(\dot{\Phi}_{R}+\dot{\Phi}_{L}\right)}{2}, \quad \mu=\frac{r\left(\dot{\Phi}_{R}-\dot{\Phi}_{L}\right)}{2 b},
$$

where $\dot{\Phi}_{R}, \dot{\Phi}_{L}$ are the angular velocities of the right and left driving wheel about the wheel shaft, respectively.

Hence, the kinematics of the WMR is written as follows [7]

$$
\left\{\begin{array}{l}
\dot{x}_{M}=\Theta \cos \theta \\
\dot{y}_{M}=\Theta \sin \theta \\
\dot{\theta}=\mu
\end{array}\right.
$$

On the other hand, when the WMR moves in the presence of slip between the wheels and the floor, (1) - (2) are no longer true. Now, let $\gamma_{R}$ and $\gamma_{L}$ denote the coordinates of the longitudinal slip of the right and left driving wheel, respectively (see Figure 1). Similarly, $\eta$ denotes the coordinate of the lateral slip along the wheel shaft. In this case, the actual linear velocity of the WMR along the longitudinal direction is shown as follows [14]

$$
\Omega=\frac{r\left(\dot{\Phi}_{R}+\dot{\Phi}_{L}\right)}{2}+\frac{\dot{\gamma}_{R}+\dot{\gamma}_{L}}{2}=\Theta+\frac{\dot{\gamma}_{R}+\dot{\gamma}_{L}}{2} .
$$

The actual angular velocity of the WMR is computed as follows [14]

$$
\omega=\frac{r\left(\dot{\Phi}_{R}-\dot{\Phi}_{L}\right)}{2 b}+\frac{\dot{\gamma}_{R}-\dot{\gamma}_{L}}{2 b}=\mu+\vartheta, \text { with } \vartheta=\frac{\dot{\gamma}_{R}-\dot{\gamma}_{L}}{2 b} .
$$

Thus, the kinematic model of this WMR can be written in terms of the coordinates of $\mathrm{M}$ as follows [14]

$$
\left\{\begin{array}{l}
\dot{x}_{M}=\Omega \cos \theta-\dot{\eta} \sin \theta \\
\dot{y}_{M}=\Omega \sin \theta+\dot{\eta} \cos \theta \\
\dot{\theta}=\omega
\end{array}\right.
$$

Due to the wheel slip, the perturbed nonholonomic constrain equations can be written as follows [16] 


$$
\begin{gathered}
\dot{\gamma}_{R}=-r \dot{\Phi}_{R}+\dot{x}_{M} \cos \theta+\dot{y}_{M} \sin \theta+b \omega, \\
\dot{\gamma}_{L}=-r \dot{\Phi}_{L}+\dot{x}_{M} \cos \theta+\dot{y}_{M} \sin \theta-b \omega \\
\dot{\eta}=-\dot{x}_{M} \sin \theta+\dot{y}_{M} \cos \theta
\end{gathered}
$$
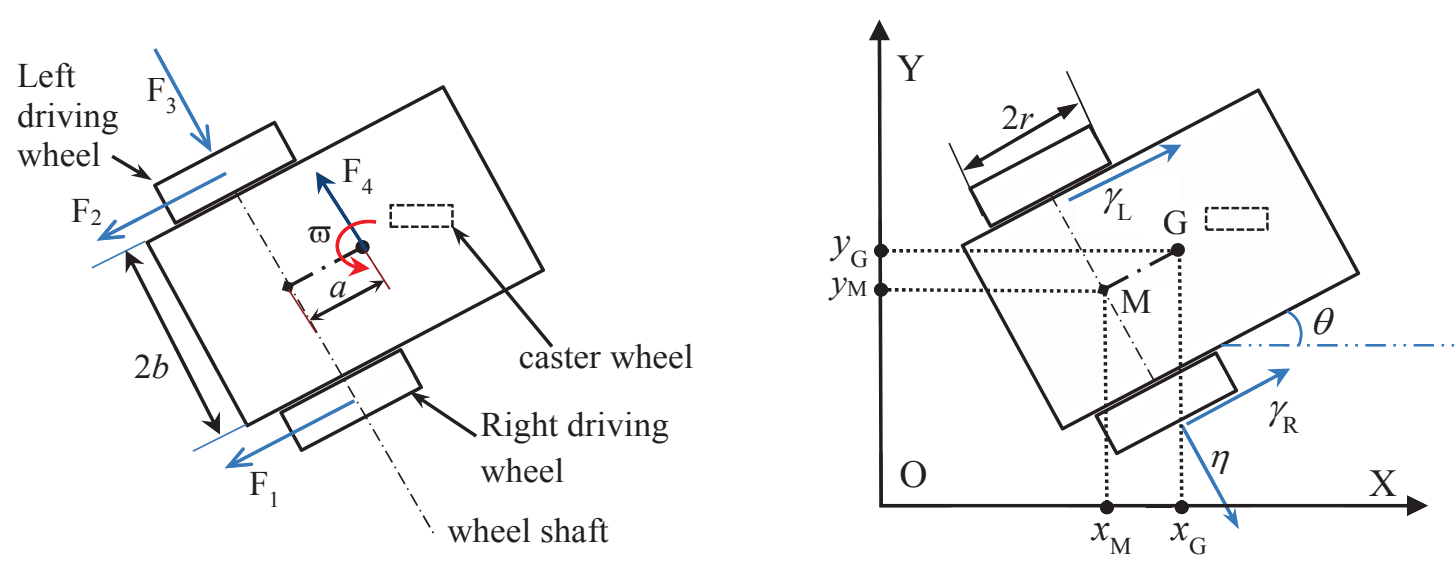

Figure 1. The nonholonomic WMR subjected to the wheel slip

\subsection{Dynamic model of the WMR with wheel slip}

The derivatives with respect to time of the coordinates of the mass center, $G$, are computed as follows

$$
\begin{aligned}
& \dot{x}_{G}=\Omega \cos \theta-\dot{\eta} \sin \theta-a \omega \sin \theta, \\
& \dot{y}_{G}=\Omega \sin \theta+\dot{\eta} \cos \theta+a \omega \cos \theta .
\end{aligned}
$$

Let $m_{G}$ be the mass of the platform of the WMR without the driving wheels. $I_{G}$ is the moment of inertia of this platform about the vertical axis through $G$. The kinetic energy of this platform is computed as follows [8]

$$
K_{G}=\frac{1}{2} m_{G}\left(\dot{x}_{G}^{2}+\dot{y}_{G}^{2}\right)+\frac{1}{2} I_{G} \omega^{2} .
$$

The kinetic energies of the right and left driving wheel are computed, respectively, as follows [8]

$$
\begin{aligned}
& K_{R}=\frac{1}{2} m_{W}\left[\left(r \dot{\Phi}_{R}+\dot{\gamma}_{R}\right)^{2}+\dot{\eta}^{2}\right]+\frac{1}{2} I_{W} \dot{\Phi}_{R}^{2}+\frac{1}{2} I_{D} \omega^{2}, \\
& K_{L}=\frac{1}{2} m_{W}\left[\left(r \dot{\Phi}_{L}+\dot{\gamma}_{L}\right)^{2}+\dot{\eta}^{2}\right]+\frac{1}{2} I_{W} \dot{\Phi}_{L}^{2}+\frac{1}{2} I_{D} \omega^{2},
\end{aligned}
$$


where $I_{W}$ or $I_{D}$ respectively is the inertial moment of each driving wheel about its rotational and diameter (vertical) axis.

The total kinetic energy of the whole system is

$$
K=K_{G}+K_{L}+K_{R}
$$

Let $\mathbf{q}=\left[x_{G}, y_{G}, \theta, \eta, \gamma_{R}, \gamma_{L}, \Phi_{R}, \Phi_{L}\right]^{T}$ be the Lagrange coordinate vector, the perturbed nonholonomic constraint equations (6), (7), and (8) can be rewritten as follows

$$
\mathbf{A}(\mathbf{q}) \dot{\mathbf{q}}=\mathbf{0} \text { where } \mathbf{A}(\mathbf{q})=\left[\begin{array}{llllllll}
\cos \theta & \sin \theta & b & 0 & -1 & 0 & -r & 0 \\
\cos \theta & \sin \theta & -b & 0 & 0 & -1 & 0 & -r \\
-\sin \theta & \cos \theta & a & -1 & 0 & 0 & 0 & 0
\end{array}\right]
$$

The potential energy of the whole system always equals to zero, so its Lagrange function is $L=K$. The Lagrange equation can be written in the following form [7]

$$
\frac{d}{d t}\left(\frac{\partial L}{\partial \dot{\mathbf{q}}}\right)-\frac{\partial L}{\partial \mathbf{q}}+\overline{\boldsymbol{\tau}}_{d}=\mathbf{N} \tau+\mathbf{A}^{T} \boldsymbol{\lambda}
$$

where $\boldsymbol{\lambda}=\left[\lambda_{1}, \lambda_{2}, \lambda_{3}\right]^{T}$ is the vector of Lagrange multipliers which are considered as constraint forces acting on the WMR so that its motion satisfies the nonholonomic constraint (15). $\boldsymbol{\tau}=\left[\tau_{R}, \tau_{L}\right]^{T}$ is the input vector with $\tau_{R}$ and $\tau_{L}$ being the torques at the right and left driving wheel about the wheel shaft, respectively. $\overline{\boldsymbol{\tau}}_{d}$ is a vector illustrating both model uncertainties such as unstructured unmodelled dynamics and unknown bounded disturbances namely unknown external forces as $\mathrm{F}_{1}$, $\mathrm{F}_{2}, \mathrm{~F}_{3}, \mathrm{~F}_{4}, \varpi$ (see Figure 1). $\mathbf{N}$ is the input transformation matrix. Solving this Lagrange equation, the dynamic equation of the whole system can be represented by

$$
\overline{\mathbf{M}} \ddot{\mathbf{q}}+\overline{\boldsymbol{\tau}}_{d}=\mathbf{N} \tau+\mathbf{A}(\mathbf{q})^{T} \boldsymbol{\lambda},
$$

where

$$
\begin{gathered}
\overline{\mathbf{M}}=\left[\begin{array}{llllllll}
m_{\mathbf{G}} & 0 & 0 & 0 & 0 & 0 & 0 & 0 \\
0 & m_{G} & 0 & 0 & 0 & 0 & 0 & 0 \\
0 & 0 & I_{G}+2 I_{D} & 0 & 0 & 0 & 0 & 0 \\
0 & 0 & 0 & 2 m_{W} & 0 & 0 & 0 & 0 \\
0 & 0 & 0 & 0 & m_{W} & 0 & m_{W} r & 0 \\
0 & 0 & 0 & 0 & 0 & m_{W} & 0 & m_{W} r \\
0 & 0 & 0 & 0 & m_{W} r & 0 & m_{W} r^{2}+I_{W} & 0 \\
0 & 0 & 0 & 0 & 0 & m_{W} r & 0 & m_{W} r^{2}+I_{W}
\end{array}\right] \\
\\
\mathbf{N}=\left[\begin{array}{ll}
0 & 0 \\
0 & 0 \\
0 & 0 \\
0 & 0 \\
0 & 0 \\
0 & 0 \\
1 & 0 \\
0 & 1
\end{array}\right] .
\end{gathered}
$$


Alternatively, it is easy to achieve the following equation [14]

$$
\dot{\mathbf{q}}=\mathbf{S}_{1}(\mathbf{q}) \mathbf{v}+\mathbf{S}_{2}(\mathbf{q}) \dot{\gamma}+\mathbf{S}_{3}(\mathbf{q}) \dot{\eta},
$$

where $\mathbf{v}=\left[\dot{\Phi}_{R}, \dot{\Phi}_{L}\right]^{T}, \boldsymbol{\gamma}=\left[\gamma_{R}, \gamma_{L}\right]^{T}, \mathbf{S}_{1}(\mathbf{q}), \mathbf{S}_{2}(\mathbf{q}), \mathbf{S}_{3}(\mathbf{q})$ are expressed by

$$
\begin{aligned}
& \mathbf{S}_{1}=\left[\begin{array}{ll}
\left(\frac{r}{2} \cos \theta-\frac{a r}{2 b} \sin \theta\right) & \left(\frac{r}{2} \cos \theta+\frac{a r}{2 b} \sin \theta\right) \\
\left(\frac{r}{2} \sin \theta+\frac{a r}{2 b} \cos \theta\right) & \left(\frac{r}{2} \sin \theta-\frac{a r}{2 b} \cos \theta\right) \\
\frac{r}{2 b} & -\frac{r}{2 b} \\
0 & 0 \\
0 & 0 \\
0 & 0 \\
1 & 0 \\
0 & 1
\end{array}\right], \\
& \mathbf{S}_{2}=\left[\begin{array}{ll}
\left(\frac{1}{2} \cos \theta-\frac{a}{2 b} \sin \theta\right) & \left(\frac{1}{2} \cos \theta+\frac{a}{2 b} \sin \theta\right) \\
\left(\frac{1}{2} \sin \theta+\frac{a}{2 b} \cos \theta\right) & \left(\frac{1}{2} \sin \theta-\frac{a}{2 b} \cos \theta\right) \\
\frac{1}{2 b} & -\frac{1}{2 b} \\
0 & 0 \\
1 & 0 \\
0 & 1 \\
0 & 0 \\
0 & 0
\end{array}\right] \\
& \mathbf{S}_{3}=\left[\begin{array}{llllllll}
-\sin \theta & \cos \theta & 0 & 1 & 0 & 0 & 0 & 0
\end{array}\right]^{T} .
\end{aligned}
$$

Next, taking the time derivative of (18), we obtain

$$
\ddot{\mathbf{q}}=\dot{\mathbf{S}}_{1}(\mathbf{q}) \mathbf{v}+\mathbf{S}_{1}(\mathbf{q}) \dot{\mathbf{v}}+\mathbf{S}_{2}(\mathbf{q}) \ddot{\gamma}+\dot{\mathbf{S}}_{2}(\mathbf{q}) \dot{\gamma}+\dot{\mathbf{S}}_{3}(\mathbf{q}) \dot{\eta}+\mathbf{S}_{3}(\mathbf{q}) \ddot{\eta},
$$

$\mathbf{S}_{1}^{T}(\mathbf{q}) \mathbf{A}^{T}(\mathbf{q})=\mathbf{0}_{2 \times 3}, \quad \mathbf{S}_{1}^{T}(\mathbf{q}) \ddot{\mathbf{S}}_{1}(\mathbf{q})=\mathbf{0}_{2 \times 2}, \quad \mathbf{S}_{1}^{T}(\mathbf{q}) \mathbf{N}=\mathbf{I}_{2 \times 2}$, where $\mathbf{I}_{i \times j}$ is an unit $i \times j$ matrix, and $\mathbf{0}_{i \times j}$ is a zero $i \times j$ matrix.

Substituting (19) into (17), and then pre-multiplying the both sides of the new equation by $\mathbf{S}_{1}^{T}(\mathbf{q})$, we get

$$
\mathbf{M} \dot{\mathbf{v}}+\mathbf{B v}+\overline{\mathbf{B}} \mathbf{v}+\mathbf{Q} \ddot{\gamma}+\mathbf{C} \omega \dot{\eta}+\mathbf{G} \ddot{\eta}+\tau_{d}=\boldsymbol{\tau}
$$

where $\overline{\boldsymbol{\tau}}_{d}=\mathbf{S}_{1}(\mathbf{q}) \overline{\boldsymbol{\tau}}_{d}$

$$
\begin{aligned}
\mathbf{M}=\mathbf{S}_{1}^{T} \overline{\mathbf{M}} \mathbf{S}_{1}= & {\left[\begin{array}{ll}
m_{11} & m_{12} \\
m_{12} & m_{11}
\end{array}\right], \quad \mathbf{Q}=\mathbf{S}_{1}^{T} \overline{\mathbf{M}} \mathbf{S}_{2}=\left[\begin{array}{ll}
Q_{11} & Q_{12} \\
Q_{12} & Q_{11}
\end{array}\right], \mathbf{C} \omega=\mathbf{S}_{1}^{T} \mathbf{M} \dot{\mathbf{S}}_{3}=m_{G} \frac{r}{2}\left[\begin{array}{l}
1 \\
1
\end{array}\right] \omega } \\
& m_{11}=m_{G}\left(\frac{r^{2}}{4}+\frac{a^{2} r^{2}}{4 b^{2}}\right)+\frac{r^{2}}{4 b^{2}}\left(I_{G}+2 I_{D}\right)+m_{W} r^{2}+I_{W}
\end{aligned}
$$




$$
\begin{gathered}
m_{12}=m_{G}\left(\frac{r^{2}}{4}-\frac{a^{2} r^{2}}{4 b^{2}}\right)-\frac{r^{2}}{4 b^{2}}\left(I_{G}+2 I_{D}\right), \\
\mathbf{Q}_{11}=m_{G} \frac{r}{4}\left(1+\frac{a^{2}}{b^{2}}\right)+\frac{r}{4 b}\left(I_{G}+2 I_{D}\right), \quad \mathbf{Q}_{12}=m_{G} \frac{r}{4}\left(1-\frac{a^{2}}{b^{2}}\right)-\frac{r}{4 b}\left(I_{G}+2 I_{D}\right), \\
\mathbf{G}=\mathbf{S}_{1}^{T} \mathbf{M S}_{3}=m_{G} \frac{a r}{2 b}\left[\begin{array}{ll}
1 \\
-1
\end{array}\right], \quad \mathbf{B}=\mathbf{S}_{1}^{T} \mathbf{M} \dot{\mathbf{S}}_{1}=m_{G} \frac{a r^{2}}{2 b} \mu\left[\begin{array}{ll}
0 & 1 \\
-1 & 0
\end{array}\right], \\
\text { and } \overline{\mathbf{B}}=\mathbf{S}_{1}^{T} \mathbf{M} \dot{\mathbf{S}}_{1}=m_{G} \frac{a r^{2}}{2 b} \vartheta\left[\begin{array}{ll}
0 & 1 \\
-1 & 0
\end{array}\right], \text { with } \mu=\frac{\dot{\Phi}_{R}-\dot{\Phi}_{L}}{2}, \vartheta=\frac{\dot{\gamma}_{R}-\dot{\gamma}_{L}}{2} .
\end{gathered}
$$

\section{CONTROL DESIGNING}

\subsection{Problem Statement}

Let $D\left(x_{D}, y_{D}\right)$ be a target which is moving in a known desired trajectory (see Figure 2). Without loss of generality, the motion equation of $D$ can be supposed as follows

$$
\left\{\begin{array}{l}
x_{D}=T_{D} \cdot t-R \cos (v \cdot t)+x_{0} \\
y_{D}=\beta T_{D} \cdot t+R \sin (v \cdot t)+y_{0},
\end{array}\right.
$$

where $\beta, T_{D}, R, v, x_{0}, y_{0}$ are constant parameters, and time $t$ varies from zero to infinity.

We assume that the tool location is at point $P$. So, the requirement of the position tracking control problem is to control the WMR so that $P$ has to track $D$ with the position tracking errors being uniformly ultimately bounded.

Remark 1. In Figure 2, we denote $\left(x_{P}, y_{P}\right)$ as the position of $P$. Let $\left(x_{P}, y_{P}, \theta\right)$ be the actual posture of the WMR, and $\left(x_{P d}, y_{P d}, \theta_{d}\right)$ be the desired one of the WMR. The presence of both the longitudinal and lateral slips makes it impossible to control the WMR in the way that the actual posture $\left(x_{P}, y_{P}, \theta\right)$ tracks the desired one $\left(x_{P d}, y_{P d}, \theta_{d}\right)$ with an arbitrarily good tracking performance. Instead of this, it is fully possible to control the WMR with the purpose of making the actual position $\left(x_{P}, y_{P}\right)$ track the desired one $\left(x_{P d}, y_{P d}\right)$ with an arbitrarily good tracking performance.

\subsection{Describing the vector of position tracking errors and the the vector of filtered tracking errors}

Let $\mathrm{O}-\mathrm{XY}$ be the global coordinate system, M-XY be the body coordinate system which is attached to the platform of the WMR (see Figure 2). The coordinate of the target is represented in $\mathrm{M}-\mathrm{XY}$ as follows [14]

$$
\boldsymbol{\zeta}=\left[\begin{array}{l}
\zeta_{1} \\
\zeta_{2}
\end{array}\right]=\left[\begin{array}{ll}
\cos \theta & \sin \theta \\
-\sin \theta & \cos \theta
\end{array}\right]\left[\begin{array}{l}
x_{D}-x_{M} \\
y_{D}-y_{M}
\end{array}\right] .
$$

Taking the second order derivative with respect to time of (22) yields [14]

$$
\ddot{\boldsymbol{\zeta}}=-\mathbf{h} \dot{\mathbf{v}}+\boldsymbol{\Psi}_{1}+\boldsymbol{\Psi}_{2},
$$


where $\mathbf{h}=\left[\begin{array}{ll}\left(\zeta_{2} \frac{1}{b}-1\right) \frac{r}{2} & -\left(\zeta_{2} \frac{1}{b}+1\right) \frac{r}{2} \\ -\zeta_{1} \frac{r}{2 b} & \zeta_{1} \frac{r}{2 b}\end{array}\right]$, and $\boldsymbol{\Psi}_{1}, \boldsymbol{\Psi}_{2}$ are nonlinear components revealed as follows

$$
\begin{gathered}
\boldsymbol{\Psi}_{1}=\mathbf{\mathbf { h }} \mathbf{v}+\left[\begin{array}{c}
\ddot{x}_{D} \cos \theta+\ddot{y}_{D} \sin \theta-\dot{x}_{D} \mu \sin \theta+\dot{y}_{D} \mu \cos \theta \\
-\ddot{x}_{D} \sin \theta+\ddot{y}_{D} \cos \theta-\dot{x}_{D} \mu \cos \theta-\dot{y}_{D} \mu \sin \theta
\end{array}\right], \\
\boldsymbol{\Psi}_{2}=\left[\begin{array}{l}
-\ddot{\chi}-\dot{x}_{D} \vartheta \sin \theta+\dot{y}_{D} \vartheta \cos \theta \\
-\ddot{\eta}-\dot{x}_{D} \vartheta \cos \theta-\dot{y}_{D} \vartheta \sin \theta
\end{array}\right],
\end{gathered}
$$

where $\mu=\frac{r\left(\dot{\Phi}_{R}-\dot{\Phi}_{L}\right)}{2}, \chi=\frac{\gamma_{R}+\gamma_{L}}{2}, \vartheta=\frac{\dot{\gamma}_{R}-\dot{\gamma}_{L}}{2}$.

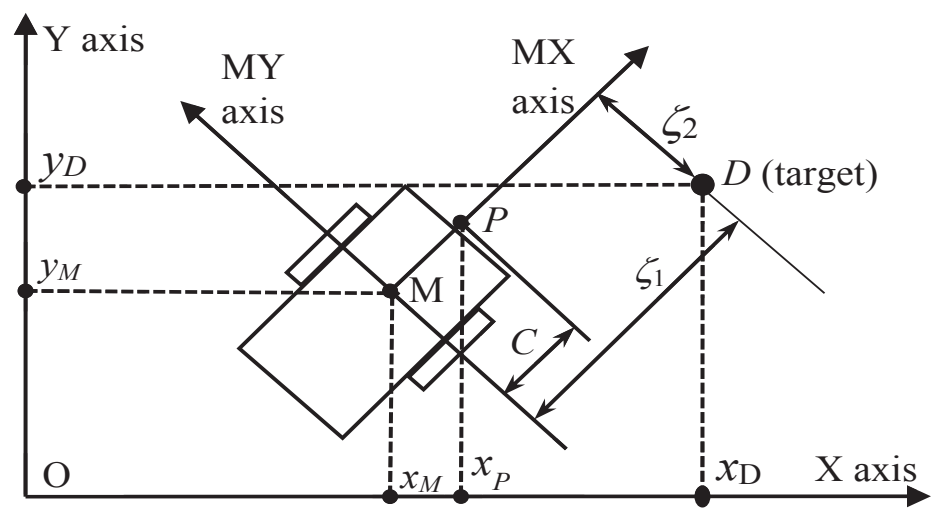

Figure 2. The coordinate of the target is represented in the body coordinate system M-XY

Remark 2. If $\zeta_{1} \neq 0$, then $\mathbf{h}$ is an invertible matrix.

Let us define the position tracking error vector as $\mathbf{e}=\left[e_{1}, e_{2}\right]^{T}=\boldsymbol{\zeta}-\boldsymbol{\zeta}_{d}$, where $\boldsymbol{\zeta}_{d}$ is the desired coordinate vector of the target in M-XY. According to the requirement of the position tracking control problem mentioned above and Figure 2, one can easily set $\boldsymbol{\zeta}_{d}=[C, 0]^{T}$.

The filtered tracking error vector is defined as follows

$$
\varphi=\dot{\mathrm{e}}+\Lambda \mathrm{e}
$$

where $\boldsymbol{\Lambda}$ is a $2 \times 2$ diagonal, constant, positive definite matrix and is chosen arbitrarily.

\subsection{Radial basis function neural network}

One cannot deny that artificial neural networks (ANN) have ability of approximating nonlinear and sufficiently smooth functions with arbitrary accuracy. Among those ANNs, the radial basis function neural network (RBFNN) is confirmed to be suitable for the purpose of approximating unknown nonlinear smooth functions. In this sub-section, the RBFNN is introduced briefly. As illustrated in Figure 3, the output of the RBFNN can be computed as follows [9] 


$$
\mathbf{y}(\mathbf{x})=\mathbf{W}^{T} \boldsymbol{\sigma}(\mathbf{x})
$$

where $\mathbf{W}$ is the weight matrix of the output layer which interconnects the hidden- with output-layer, and $\boldsymbol{\sigma}(\mathbf{x})$ is the vector of activation functions in the hidden layer, $\mathbf{x}$ is the input vector of the RBFNN.

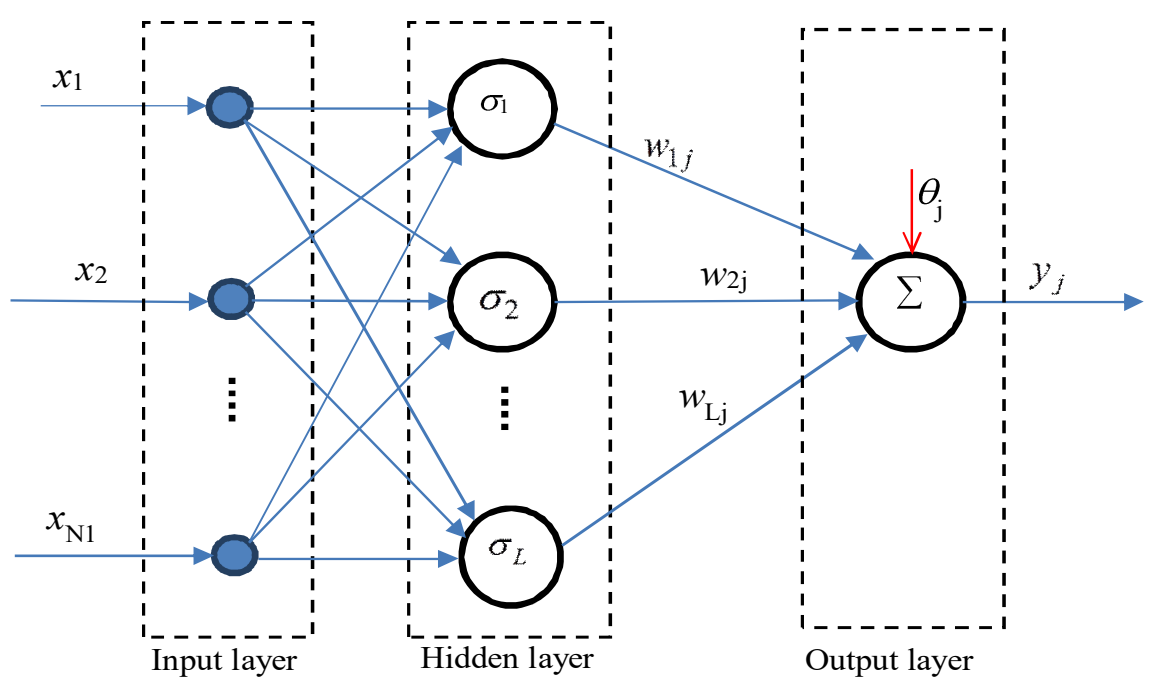

Figure 3. Structure of RBFNN

In particular, if there are $L$ neurons in the hidden layer and $N$ neurons in the output layer, then $\mathbf{W} \in \mathbf{R}^{(L+1) \times N}$ and $\boldsymbol{\sigma}(\mathbf{x}) \in \mathbf{R}^{(1+L) \times 1}$ are respectively expressed as follows

$$
\mathbf{W}=\left[\begin{array}{lllll}
\theta_{1} & \theta_{2} & \cdots & \cdots & \theta_{N} \\
w_{11} & w_{21} & \cdots & \cdots & w_{1 N} \\
w_{12} & w_{22} & \cdots & \cdots & w_{2 N} \\
\vdots & \vdots & \vdots & \ddots & \vdots \\
w_{L 1} & w_{L 2} & \cdots & \cdots & w_{L N}
\end{array}\right] \text {, and } \boldsymbol{\sigma}(\mathbf{x})=\left[\begin{array}{l}
1 \\
\sigma_{1} \\
\sigma_{2} \\
\vdots \\
\sigma_{L}
\end{array}\right] \text {, where } \theta_{i} \text { are the threshold }
$$

offsets of the output layer (see Figure 3). It is noticeable that putting 1 into the first component of $\boldsymbol{\sigma}(\mathbf{x})$ allows one to comprise the threshold vector $\left[\theta_{1}, \theta_{2}, \ldots, \theta_{L}\right]^{T}$ as the first row of $\mathbf{W}$. That is to say, any tuning of $\mathbf{W}$ consists of tuning of both the weights $w_{i j}$ of the connections from the hiddento output-layer and thresholds $\theta_{i j}$ at the output-layer.

For the activation functions of the hidden layer, the Gaussian type function is employed as follows

$$
\sigma_{i}=\exp \left(-\frac{1}{2 \rho_{i}^{2}}\left\|\mathbf{x}-\boldsymbol{\Xi}_{i}\right\|^{2}\right)
$$

where $\boldsymbol{\Xi}_{i}$ and $\rho_{i}$ are the center and width of the $i$-th hidden neuron, $i=1,2, \ldots, L$. 
Given any bounded and continuous function $\mathbf{f}(\mathbf{x}): R^{M} \rightarrow R^{N}$, there is an ideal matrix $\mathbf{W}$ so that we can express $\mathbf{f}(\mathbf{x})$ as follows [9]

$$
\mathbf{f}(\mathbf{x})=\mathbf{y}(\mathbf{x})+\varepsilon=\mathbf{W}^{T} \boldsymbol{\sigma}(\mathbf{x})+\varepsilon,
$$

where $\varepsilon \in \mathbf{R}^{N \times 1}$ is a vector illustrating reconstruction errors.

Assumption 1. With a large enough number $L$ of the hidden-layer neurons, there exists $b_{\varepsilon}$ such that $\|\varepsilon\| \leq b_{\varepsilon}$.

An approximation of $\mathbf{f}(\mathbf{x})$ is revealed by $\hat{\mathbf{f}}(\mathbf{x})=\hat{\mathbf{W}}^{T} \boldsymbol{\sigma}(\mathbf{x})$, where $\hat{\mathbf{W}}$ is an estimation matrix of $\mathbf{W}$ and is provided by an online weight tuning algorithm to be discussed subsequently.

The function approximation error vector is computed by

$$
\tilde{\mathbf{f}}=\mathbf{f}(\mathbf{x})-\hat{\mathbf{f}}(\mathbf{x})=\mathbf{W}^{T} \boldsymbol{\sigma}(\mathbf{x})+\boldsymbol{\varepsilon}-\hat{\mathbf{W}}^{T} \boldsymbol{\sigma}(\mathbf{x}) .
$$

\subsection{Controller structure and error system dynamics}

In (23), since directly depending on the accelerations and velocities of the wheel slips which are not measured in this work, $\boldsymbol{\Psi}_{2}$ is unknown. Therefore, let us define an auxiliary variable which can be measured easily as follows

$$
\dot{\mathbf{v}}_{c}=\mathbf{h}^{-1}\left(-\dot{\boldsymbol{\zeta}}_{d}+\Lambda \dot{\mathbf{e}}+\Psi_{1}\right) .
$$

Alternatively, one can rewrite (20) as follows

$$
\mathrm{M} \dot{\mathbf{v}}=\boldsymbol{\tau}-\mathrm{Bv}-\mathbf{d}
$$

where $\mathbf{d}=\overline{\mathbf{B v}}+\mathbf{Q} \ddot{\gamma}+\mathbf{C} \omega \dot{\eta}+\mathbf{G} \ddot{\eta}+\boldsymbol{\tau}_{d}$.

Subtracting $\mathbf{M} \dot{\mathbf{v}}_{c}$ from both of the sides of (29) and then combining the result and (23), (24), and (28) leads to

$$
-\mathbf{M h}^{-1} \dot{\boldsymbol{\varphi}}=\boldsymbol{\tau}-\mathbf{M} \dot{\mathbf{v}}_{c}-\mathbf{B v}-\mathbf{d}-\mathbf{M h}^{-1} \Psi_{2} .
$$

However, it is difficult to exactly know the parameters of the dynamic model of this WMR such as mass, moments of inertia, etc. Consequently, it is impossible to precisely describe all expressions including these quantities. For this reason, let $\hat{\mathbf{M}}$ be an approximation of $\mathbf{M}$. One can rewrite (30) as follows

$$
-\hat{\mathbf{M}} \mathbf{h}^{-1} \dot{\varphi}=\boldsymbol{\tau}-\tilde{\mathbf{M}} \mathbf{h}^{-1} \dot{\varphi}-\mathbf{M} \dot{\mathbf{v}}_{c}-\mathbf{B v}-\mathbf{d}-\mathbf{M h}^{-1} \mathbf{\Psi}_{2},
$$

where $\tilde{\mathbf{M}}=\mathbf{M}-\hat{\mathbf{M}}$.

Remark 3. It should be noted that both $\mathbf{M}, \hat{\mathbf{M}}$ are always symmetric, invertible, positive definite matrices.

Multiplying both of the sides of (31) by $-\mathbf{h} \hat{\mathbf{M}}^{-1}$ yields

$$
\dot{\varphi}=-\mathbf{h} \hat{\mathbf{M}}^{-1} \boldsymbol{\tau}+\mathbf{f}(\mathbf{x})+\Delta,
$$

where $\mathbf{f}(\mathbf{x})=\mathbf{h} \hat{\mathbf{M}}^{-1}\left(\mathbf{M} \dot{\mathbf{v}}_{c}+\mathbf{B v}\right)$, and $\boldsymbol{\Delta}=\mathbf{h} \hat{\mathbf{M}}^{-1}\left(\tilde{\mathbf{M}} \mathbf{h}^{-1} \dot{\boldsymbol{\varphi}}+\mathbf{d}+\mathbf{M h}^{-1} \mathbf{\Psi}_{2}\right)$. 
The vector $\mathbf{x}$ demanded so as to calculate $\mathbf{f}(\mathbf{x})$ can be determined by $\mathbf{x}=\left[\begin{array}{ll}\mathbf{v}^{T} & \dot{\mathbf{v}}_{c}^{T}\end{array}\right]^{T}$. Clearly, $\mathbf{x}$ can be measured easily.

In (32), $\mathbf{f}(\mathbf{x})$ can be approximated by the RBFNN described by (26). Therefore, one can choose a torque-computing control law as follows

$$
\tau=\hat{\mathbf{M}} \mathbf{h}^{-1}\left(\mathbf{K} \varphi+\Gamma \frac{\varphi}{\|\varphi\|}+\hat{\mathbf{f}}\right)
$$

where $\mathbf{K}$ is a $2 \times 2$ diagonal, constant, positive definite matrix and can be chosen arbitrarily. $\hat{\mathbf{f}}$ is the output of the RBFNN described by (??) and is an estimation of $\mathbf{f}(\mathbf{x})$ in (32). $\Gamma \frac{\varphi}{\|\varphi\|}$ is a robust term which is used to overcome the model uncertainties, unknown external disturbances, and the unknown wheel slips described by $\boldsymbol{\Delta}$ in (32). $\Gamma$ is a positive constant value and can be selected arbitrarily.

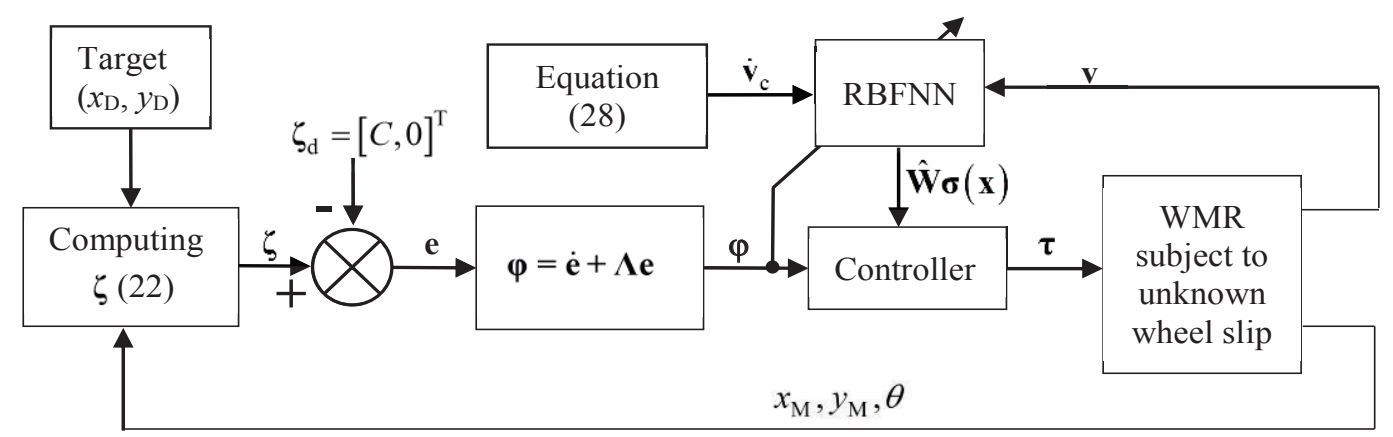

Figure 4. Scheme of control system

Combining (33) and (32) yields:

$$
\dot{\varphi}=-\mathbf{K} \varphi-\Gamma \frac{\varphi}{\|\varphi\|}+\tilde{\mathbf{f}}(\mathbf{x})+\Delta
$$

where $\tilde{\mathbf{f}}(\mathbf{x})=\mathbf{f}(\mathbf{x})-\hat{\mathbf{f}}(\mathbf{x})$ is represented in $(27)$.

Substitution of (27) into (34) makes the filtered tracking error dynamics become

$$
\dot{\varphi}=-\mathbf{K} \varphi-\Gamma \frac{\varphi}{\|\varphi\|}+\tilde{\mathbf{W}}^{T} \boldsymbol{\sigma}+\varepsilon+\Delta
$$

where $\tilde{\mathbf{W}}$ as the weight deviation by $\tilde{\mathbf{W}}=\mathbf{W}-\hat{\mathbf{W}}$.

With such structure of the RBFNN, a suitable tuning rule for the weights should be determined to train the RBFNN. In this work, let us propose the online weight tuning algorithm for the RBFNN as follows

$$
\dot{\hat{\mathbf{W}}}=\mathbf{H} \sigma \varphi^{T}
$$

where $\mathbf{H}$ is a $(L+1) \times(L+1)$ positive definition constant matrix. $\mathbf{H}$ can be chosen arbitrarily.

Assumption 2. It is assumed that $\Delta$ is bounded. Let $b_{\Delta}$ be the upper bound of $\Delta$. It means that $\|\Delta\| \leq b_{\Delta}$. 
Theorem 1. Let us consider the WMR subjected to the wheel slips with the dynamics shown as (20). Let assumptions 1-3 hold. Let us choose the control input as (33) and the scheme of the whole system as Figure 4. Let us provide the weight tuning algorithm by (36). By doing this, the filtered tracking error vector $\varphi$ uniformly ultimately converge to an arbitrarily small neighborhood of the origin. Moreover, $\boldsymbol{\varphi}$ can be made be as small as possible by choosing $\mathbf{K}$ and $\Gamma$ to be suitable.

Proof.

Let us define a Lyapunov candidate function as follows

$$
V=\frac{1}{2} \boldsymbol{\varphi}^{\mathbf{T}} \boldsymbol{\varphi}+\frac{1}{2} \operatorname{tr}\left(\tilde{\mathbf{W}}^{T} \mathbf{H}^{-1} \tilde{\mathbf{W}}\right)
$$

where $\operatorname{tr}($.$) is the trace of matrix.$

Taking the first derivative with respective to time yields

$$
\dot{V}=\varphi^{T} \dot{\boldsymbol{\varphi}}+\operatorname{tr}\left(\tilde{\mathbf{W}}^{T} \mathbf{H}^{-1} \dot{\tilde{\mathbf{W}}}\right)
$$

Due to, (38) becomes

$$
\dot{V}=\varphi^{T} \dot{\boldsymbol{\varphi}}-\operatorname{tr}\left(\tilde{\mathbf{W}}^{T} \mathbf{H}^{-1} \dot{\hat{\mathbf{W}}}\right) .
$$

Substitution of (35) and (36) into (39) results in

$$
\dot{V}=\boldsymbol{\varphi}^{T}\left[-\mathbf{K} \boldsymbol{\varphi}-\boldsymbol{\Gamma} \frac{\boldsymbol{\varphi}}{\|\boldsymbol{\varphi}\|}+\tilde{\mathbf{W}}^{T} \boldsymbol{\sigma}+\boldsymbol{\varepsilon}+\boldsymbol{\Delta}\right]-\operatorname{tr}\left[\tilde{\mathbf{W}}^{T} \boldsymbol{\sigma} \boldsymbol{\varphi}^{T}\right] .
$$

Due to $\boldsymbol{\varphi}^{T} \tilde{\mathbf{W}}^{T} \boldsymbol{\sigma}=\operatorname{tr}\left(\tilde{\mathbf{W}}^{T} \boldsymbol{\sigma} \boldsymbol{\varphi}^{T}\right),(40)$ becomes

$$
\dot{V}=-\varphi^{T} \mathbf{K} \varphi-\Gamma\|\varphi\|-\varphi^{T} \varepsilon-\varphi^{T} \Delta .
$$

According to Assumption 1, Assumption 2, one can easily obtain the following inequality

$$
\dot{V} \leq-\|\varphi\|\left[K_{\min }\|\varphi\|+\Gamma-b_{\varepsilon}-b_{\Delta}\right],
$$

where $K_{\min }$ is the minimum singular value of $\mathbf{K}$.

Observing (42) reveals that $\dot{V}$ is guaranteed to be negative definiteness as long as the term in the braces is positive. This term is assured to be positive as long as

$$
K_{\min }\|\varphi\|+\Gamma>b_{\varepsilon}+b_{\Delta} .
$$

Therefore, applying Lyapunov criteria and LaSalle extension results in that $\varphi$ is uniformly ultimately bounded in a compact set as follows

$$
\mathbf{U}=\left\{\boldsymbol{\varphi} \mid K_{\min }\|\boldsymbol{\varphi}\|+\Gamma \leq b_{\varepsilon}+b_{\Delta}\right\} .
$$

It is remarkable to note that both $\varphi$ can be made be as small as possible by choosing $K$, and $\Gamma$ suitably. Particularly, the bigger $K$ and $\Gamma$ are, the smaller $\varphi$ is. This illustrates the uniformly ultimately bounded property of $\varphi$ in the sense of Lyapunov and LaSalle extension.

As a result of convergence of $\varphi$ to an adjustable small neighborhood of the origin, the position tracking error $\mathbf{e}$ in (24) also has converged to an adjustable small neighborhood of the origin. 


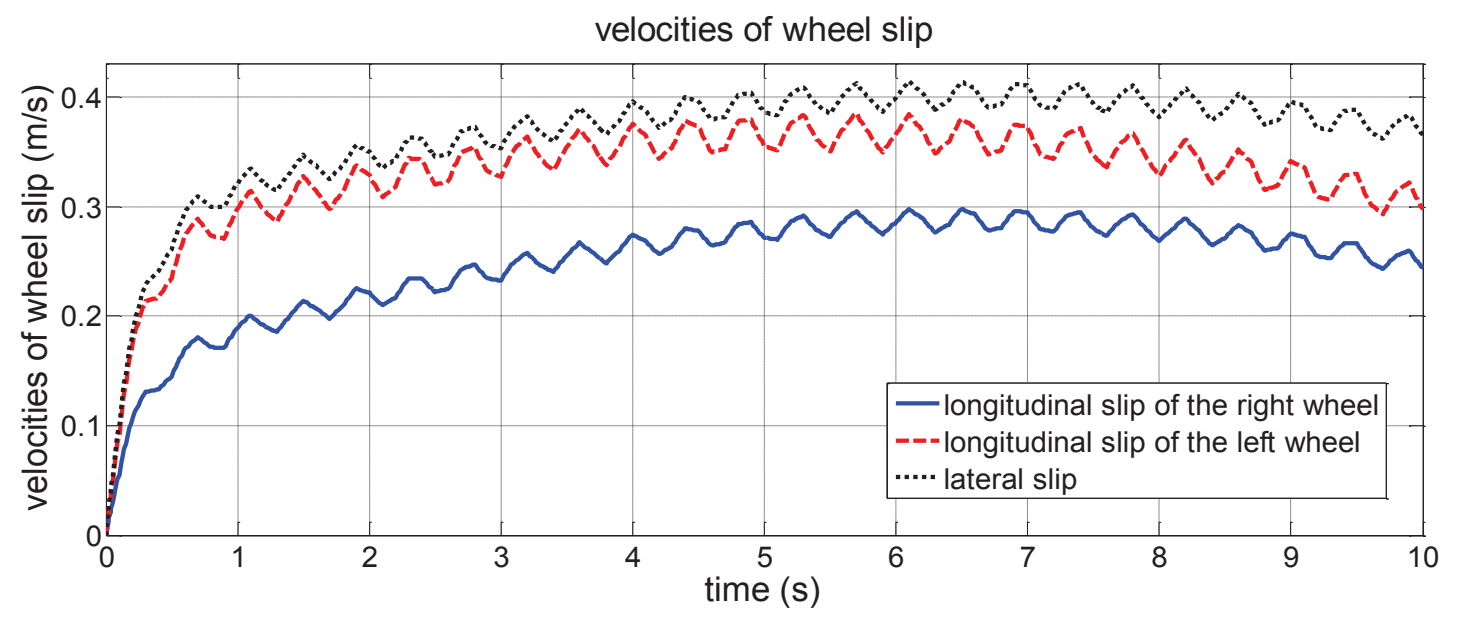

Figure 5. The evolutions of wheel slip

\section{SIMULATION AND DISCUSSION}

To verify the proposed control law, we have implemented a simulation for trajectory tracking of the WMR whose parameters is shown in Table 1 in the presence of the unknown wheel slips. In the initial condition, assume that the initial posture of $\mathrm{M}$ in the global coordinate system is $x_{M}=0(\mathrm{~m})$, $y_{M}=0(\mathrm{~m})$, and $\theta=0.1(\mathrm{rad})$. It infers that $x_{P}=C=0.5(\mathrm{~m}), y_{P}=0(\mathrm{~m})$. Furthermore, we have made a comparison between the performances of this proposed control and the control method in [14]. To represent the compensation capability of the proposed control method on the uncertainty effects, the vector of the both model uncertainties and bounded external disturbances is assumed as $\boldsymbol{\tau}_{d}=\left[\begin{array}{ll}3+\sin (0.5 t), & 2.5+\cos (0.4 t)\end{array}\right]^{T} \quad(N . m)$ and $\hat{\mathbf{M}}=0.7 \mathbf{M}$.

The control parameters were chosen as $\mathbf{K}=\operatorname{diag}([6,6]), \boldsymbol{\Lambda}=\operatorname{diag}([2,2])$. The hidden layer has 10 neurons. The weight tuning gain was set as $\mathbf{H}=\operatorname{diag}(10)_{11 \times 11}$. The initial condition of the weight matrix was chosen to random numbers in $[0,1]$ as $\hat{\mathbf{W}}_{0}=[\operatorname{rand}(0,1)]_{11 \times 2}$.

The target (point D) moved with a motion equation described as follows

$$
\left\{\begin{array}{c}
x_{D}=6-3 \cos (0.25 t) \\
y_{D}=-2-3 \sin (0.25 t)
\end{array}\right.
$$

Without loss of generality, assume that the wheel slips between the floor and the driving wheels have been illustrated as Figure 5 .

The computer simulation results were performed by Matlab/Simulink software. Obviously, in Figures 6 and 7, we can easily see that when the accelerations and velocities of unknown wheel slips have not been measured and further there existed model uncertainties as well as unknown bounded disturbances, in comparison with the tracking results and position tracking errors of the feedback linearization control method in [14], those of the proposed control method are better. In other words, the performance of the proposed control method is better than that of the feedback linearization control method.

Figure 8 has shown that the outputs of the RBFNN have been bounded.

It is apparent that the position tracking error vector, $\mathbf{e}$, in (24) has converged to an adjustable small neighborhood of the origin, so $\xi_{1}$ has converged to an adjustable small neighborhood of $C$. 
Comparison of tracking results

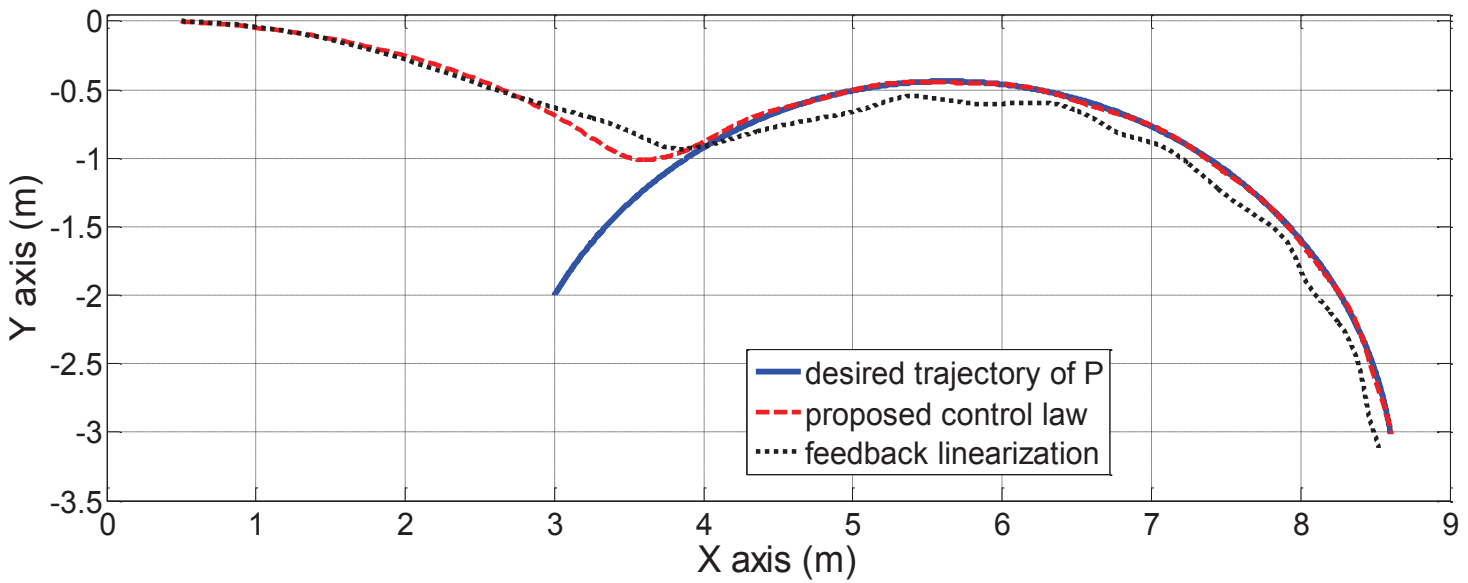

Figure 6. Comparison of tracking results
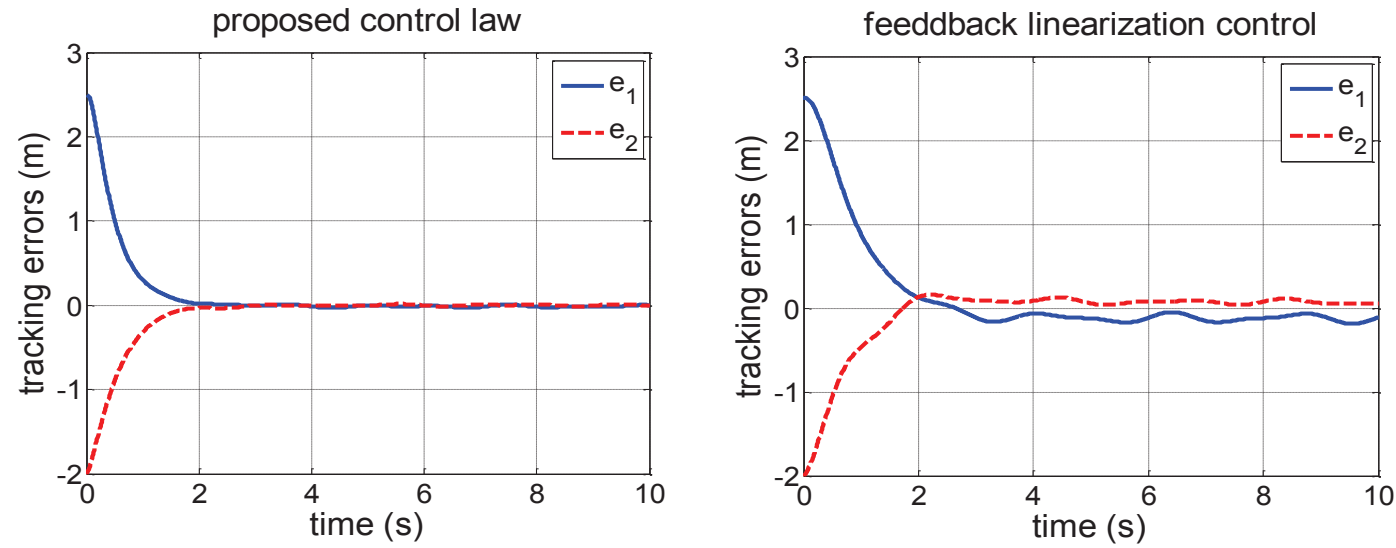

Figure 7. Comparison of tracking errors

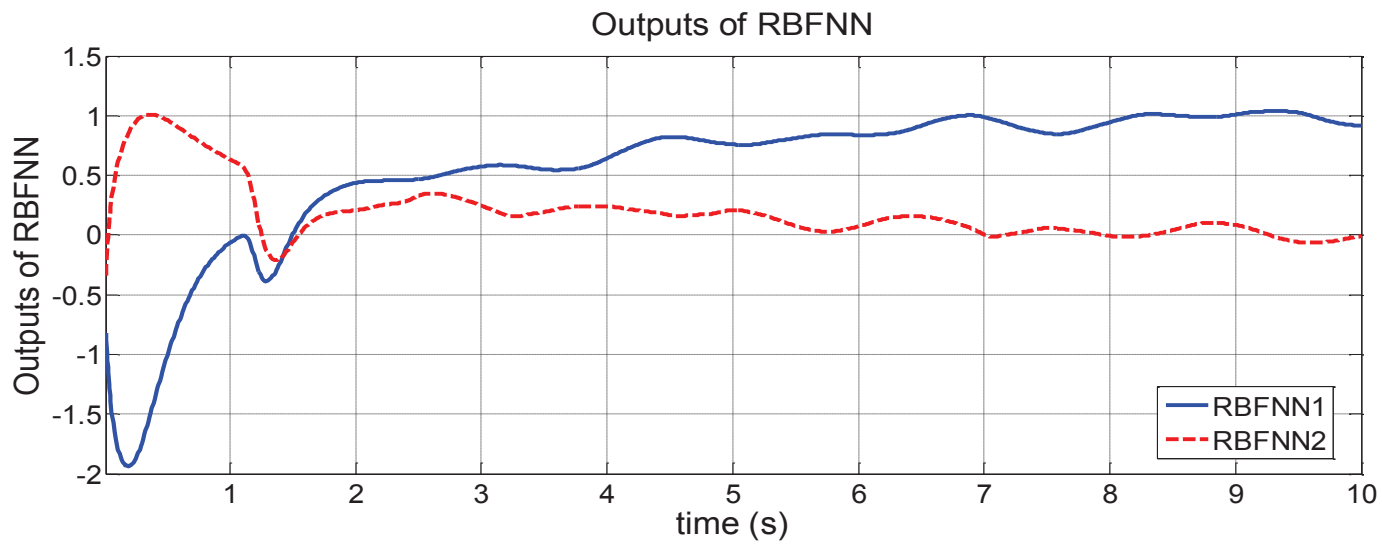

Figure 8. Outputs of RBFNN 
As a result, according to Remark 2, one can easily conclude that $\mathbf{h}$ in (28), (30), (31), and (33) is invertible.

From these simulation results, we can conclude that the proposed control method has robustness against the unknown wheel slips, the model uncertainties, and the unknown external disturbances. Furthermore, all signals in the whole closed-loop system have been bounded.

Table 1. The parameters of the WMR

\begin{tabular}{|c|c|c|}
\hline Symbol & Quantity & Value \\
\hline$m_{G}$ & The mass of the platform of the WMR & $40(\mathrm{~kg})$ \\
\hline$I_{G}$ & $\begin{array}{l}\text { The inertial moment of the platform about the vertical } \\
\text { axis through point } G \text { (Fig. } 1 \text { ) }\end{array}$ & $4\left(\mathrm{~kg} \cdot \mathrm{m}^{2}\right)$ \\
\hline$a$ & The distance between point $G$ and point $M$ (Fig. 1) & $0.2(\mathrm{~m})$ \\
\hline$C$ & The distance between point $P$ and point $M$ (Fig. 2) & $0.5(\mathrm{~m})$ \\
\hline$m_{W}$ & The mass of each wheel & $2(\mathrm{~kg})$ \\
\hline$I_{W}$ & $\begin{array}{l}\text { The inertial moment of each wheel about its rotational } \\
\text { axis }\end{array}$ & $0.1\left(\mathrm{~kg} \cdot \mathrm{m}^{2}\right)$ \\
\hline$I_{D}$ & $\begin{array}{l}\text { The inertial moment of each wheel about its diameter } \\
\text { axis }\end{array}$ & $0.05\left(\mathrm{~kg} \cdot \mathrm{m}^{2}\right)$ \\
\hline$b$ & half-distance between two the wheels & $0.3(\mathrm{~m})$ \\
\hline$r$ & The radius of each wheel & $0.15(\mathrm{~m})$ \\
\hline
\end{tabular}

\section{CONCLUSIONS}

In this work, well-known Lagrange formula was employed in order to derive both the kinematics and dynamics of the nonholonomic WMR in the presence of the unknown wheel slips. Then, the adaptive tracking controller based on the RBFNN with the online weight tuning algorithm has been developed to allow the WMR to track the desired trajectory with the desired tracking performance. The RBFNN functional approximation errors and the effect of the unknown wheel slips have been dealt with in the same way as the model uncertainties as well as the unknown external disturbances, since they all have the same influence on the closed-loop system. A priori offline train for the weights of the RBFNN was not needed since they can be initialized without difficulty. It has been shown that the convergence of the position tracking errors to an arbitrarily small neighborhood of the origin is guaranteed by the standard Lyapunov theory and LaSalle extension. The results of Matlab/Simulink simulation confirmed the effectiveness and advantage of the proposed controller.

\section{REFERENCES}

[1] H. Bach and H. Kang, "Neural network-based adaptive tracking control of mobile robots in the presence of wheel slip and external disturbance force," Neurocomputing, vol. 118, pp. $12-22$, 2016.

[2] L. C. Boon and W. Danwei, "Integrated estimation for wheeled mobile robot posture, velocities, and wheel skidding perturbations," Proc of the IEEE Int Conf on Robot and Autom, pp. 2355$2360,2007$. 
[3] C. Y. Chen, T. H. S. Li, Y. C. Yeh, and C. C. Chang, "Design and implementation of an adaptive sliding mode dynamic controller for wheeled mobile robots," Mechatronics, vol. 19, no. 2, pp. 156-166, 2009.

[4] D. Chwa, "Sliding-mode tracking control of nonholonomic wheeled mobile robots in polar coordinates," IEEE Trans. Control Syst, vol. 12, no. 4, pp. 637-644, 2004.

[5] — - "Fuzzy adaptive tracking control of wheeled mobile robots with state-dependent kinematic and dynamic disturbances," IEEE Trans. Fuzzy Syst, vol. 20, no. 3, pp. 587-593, 2012.

[6] L. Ding, H. B. Gao, Z. Q. Deng, and Z. Liu, "Slip-ratio-coordinated control of planetary exploration robots traversing over deformable rough terrain," IEEE Int Conf on Intel Robo and Syst, pp. 4958-4963, 2010.

[7] R. Fierro and F. L. Lewis, "Control of a nonholonomic mobile robot using neural networks," IEEE Trans. Neur. Netw, vol. 9, no. 4, pp. 589-600, 1998.

[8] T. Fukao, H. Nakagawa, and N. Adachi, "Adaptive tracking control of a nonholonomic mobile robot," IEEE Trans. Robot. Autom, vol. 16, no. 1, pp. 609-615, 2000.

[9] H. Gao, X. Song, L. Ding, K. Xia, and Z. D. N. Li, "Adaptive motion control of wheeled mobile robot with unknown slippage," Int. J. Control, vol. 87, no. 8, pp. 1513-1522, 2014.

[10] C. B. Low and D. Wang, "Gps-based path following control for a car-like wheeled mobile robot with skidding and slipping," IEEE Trans. Control Syst. Technol, vol. 16, no. 2, pp. 340-347, 2008 .

[11] — - "Gps-based tracking control for a car-like wheeled mobile robot with skidding and slipping," IEEE Trans. Mechatron, vol. 13, no. 4, pp. 480-484, 2008.

[12] S. A. A. Moosavian and A. Kalantari, "Experimental slip estimation for exact kinematics modeling and control of a tracked mobile robot," Proc. IEEE/RSJ Int Conf on Intel Robo and Syst, pp. 95-100, 2008.

[13] M. Seyr and S. Jakubek, "Proprioceptive navigation, "slip estimation and slip control for autonomous wheeled mobile robots," Proc. of the IEEE Conf. on Robot, Autom and Mecha, pp. $1-6,2006$.

[14] N. V. Tinh, N. T. Linh, P. T. Cat, P. M. Tuan, M. N. Anh, and N. P. Anh, "Modeling and feedback linearization control of a nonholonomic wheeled mobile robot with with longitudinal, lateral slips," IEEE Int Conf on Autom Sci and Engi, pp. 21-24, 2016.

[15] D. Wang and C. Low, "Modeling and analysis of skidding and slipping in wheeled mobile robots: Control design perspective," IEEE Trans. Robot, vol. 24, no. 3, pp. 676-687, 2008.

[16] S. J. Yoo, "Approximation-based adaptive control for a class of mobile robots with unknown skidding and slipping," Int Jour of Cont, Autom, and Syst, vol. 10, no. 4, pp. 703-710, 2012.

[17] — - "Adaptive neural tracking and obstacle avoidance of uncertain mobile robots with unknown skidding and slipping," Information Sciences, vol. 238, pp. 176-189, 2013. 